ORIGINAL ARTICLE

\title{
Prevalence of gastric precancerous lesions in Ardabil, a high incidence province for gastric adenocarcinoma in the northwest of Iran
}

\author{
R Malekzadeh, M Sotoudeh, M H Derakhshan, J Mikaeli, A Yazdanbod, S Merat, A Yoonessi, \\ M Tavangar, B Abedi Ardakani, R Sotoudehmanesh, A Pourshams, A Ali Asgari, S Doulatshahi, \\ B Ziad Alizadeh, S Arshi, A Madjidpoor, S Mir Moomen, D E Fleischer
}

J Clin Pathol 2004;57:37-42

See end of article for authors' affiliations

Correspondence to: Professor R Malekzadeh, Digestive Disease Research Centre, Shariati Hospital, North Kargar Avenue, Tehran, 14114, Iran; malek@ams.ac.ir

Accepted for publication 13 July 2003

\begin{abstract}
Background/Aims: Ardabil Province, in northwestern Iran, has the highest rate of gastric (predominantly cardia) adenocarcinoma in Iran. This study aimed to investigate the feasibility of endoscopic screening and to look for associated Helicobacter pylori infection and gastric precancerous lesions.

Methods: One thousand one hundred and five adult volunteers, residents of Ardabil and Meshkinshahr, districts, 40 years old and above were selected and invited by a simple random household canvass in rural and urban locations. Informed consents were obtained and upper gastrointestinal video endoscopy was performed to biopsy all visible lesions and standard sites in the antrum, corpus, and cardia

Results: One thousand and eleven of the invited individuals agreed to participate, including 494 men and 517 women, with a mean (SD) age of 53.32 (10.39) years. Endoscopy was well tolerated by all subjects; $96.7 \%$ of antral and $80.7 \%$ of cardia mucosal biopsies were satisfactory. The urease test or histology for $\mathrm{H}$ pylori was positive in at least $89.2 \%$ of subjects. Histological evidence of mucosal atrophy was seen in $39.3 \%$ of antral and $21.9 \%$ of cardia samples. Chronic gastritis with or without activity, reactive atypia of glandular epithelium, intestinal metaplasia, dysplasia, and cancer were found in $95.1 \%, 38.0 \%, 8.7 \%$, $0.2 \%$, and $0.3 \%$ of antral and $85.3 \%, 22.9 \%, 3.8 \%, 0.3 \%$, and $0.1 \%$ of cardiac biopsies, respectively. Conclusion: Endoscopic screening for upper gastrointestinal diseases was feasible and well tolerated in Ardabil, Iran. Most subjects showed $H$ pylori infection. Atrophic gastritis, reactive atypia, and intestinal metaplasia were common in antrum, corpus, and cardia subsites.
\end{abstract}

G astric adenocarcinoma is the second most common cause of cancer related mortality in the world. ${ }^{12}$ This tumour is also the most common gastrointestinal (GI) malignancy in Iran. ${ }^{3}$ Ardabil, a northwestern province of Iran, was reported to have the highest incidence of gastric adenocarcinoma in the whole country. According to an active cancer surveillance programme in Ardabil Province, covering a period of four years (1996-1999), this lesion constitutes $31 \%$ of all malignancies seen in that region, with an incidence rate of 49.1 and 25.4/100 000/year for men and women, respectively. ${ }^{5}$ A prospective study of patients with upper GI malignancy diagnosed in a single referral centre in the city of Ardabil during a period of one year revealed that the proximal stomach (cardia) is the most common location for malignancy in the upper GI, constituting about $50 \%$ of the cases in that series. ${ }^{6}$ Helicobacter pylori is known to be involved in the multistep process that finally leads to gastric adenocarcinoma, with estimates of attributable risk up to $75 \% .^{78}$

"Eradication of Helicobacter pylori infection is a promising option for the prevention of gastric cancer, at least in high risk regions of the world"

This organism has been classified as a member of the group 1 carcinogenic agents by the World Health Organisation. ${ }^{9}$ A recent study using an animal model has clearly shown that long term $H$ pylori infection of the gastric mucosa can progress to adenocarcinoma. ${ }^{10}$ Uemura et al showed that both intestinal and diffuse type gastric adenocarcinoma were closely associated with $H$ pylori infection. ${ }^{11}$ This evidence, together with the very low five year survival rate of gastric cancer, favours the notion that eradication of $H$ pylori infection is a promising option for the prevention of gastric cancer, at least in high risk regions of the world. There is also some evidence to support the protective effects of antioxidants, such as $\beta$ carotene and ascorbic acid, against gastric cancer, ${ }^{12}$ and the results of a recently published randomised controlled trail revealed that $H$ pylori eradication, together with supplementing the diet with antioxidants as a preventive strategy has considerable public health potential. ${ }^{13}$

During a scientific collaboration between the Digestive Disease Research Centre (DDRC) of Tehran University of Medical Sciences and Ardabil University of Medical Sciences, aimed at devising a preventive strategy for gastric cancer in Ardabil, we sought to investigate the feasibility of endoscopic screening studies and to monitor the prevalence of $H$ pylori infection and its related precancerous lesions in the residents of two major districts of the Ardabil Province.

\section{MATERIALS AND METHODS}

\section{Study population}

Our study was performed between June and September 2000 in Ardabil district, which has a total population of 435487 , and July and September 2001 in Meshkinshahr district, which has a population of 164 007. In total, 1105 households

Abbreviations: DDRC, Digestive Disease Research Centre; GI, gastrointestinal; IM, intestinal metaplasia 
were canvassed in both districts. Part of each city was selected on a random basis, incorporating the adjoining rural area lying within a distance of 5-50 km from the city limits, an area that included 17 villages in Ardabil and 19 villages in Meshkinshahr. Expert local health professionals interviewed those members of the family who were aged 40 years or more, and thoroughly explained the purpose and procedure of the study to them. One individual from the target age group in each family was randomly selected to be invited to participate in our study. If such an individual was not available, or was excluded by any of the exclusion criteria, the immediate neighbour to the right hand side was referred to for the same process. Exclusion criteria used in our study were: unwillingness to participate at any stage of the study and for any reason, inability to tolerate upper GI endoscopy for any reason decided by the health professional during the interview or the gastroenterologist in charge at the time of endoscopy, history of known gastrointestinal disease in the past, or the presence of significant benign or malignant upper GI disease. All the individuals who were willing to participate were transferred to the site of study on the appointed day.

\section{PROCEDURES}

Upon arrival at the endoscopy clinic on the appointed day, the purpose of our study and the risks and benefits of the procedures were explained to the participants for the second time and the individuals signed a written informed consent to undergo the procedures necessary for our study. The study protocol and the informed consent used for this investigation were approved by the ethics committee of the DDRC, Tehran University of Medical Sciences before the study.

Before endoscopy, each participant was interviewed by a trained interviewer (general practitioner) to obtain the necessary information by filling in a questionnaire. The questionnaire was designed to collect demographic, nutritional, and important life style information. Specific sections included questions covering the staple diet and nutritional variety, food preservation facilities, and source of drinking water. Other sections included questions about life style and habits including smoking, alcohol, and drug abuse. Pertinent history for any important health problem with emphasis on important GI symptoms was obtained. The individuals were asked about any history of upper GI malignancy in first degree relatives.

After the interview, a limited physical examination was performed including the recording of height and weight. A few drops of blood taken from the tip of a finger punctured by a lancet under disinfected conditions were collected on to a Watman No. 1 filter paper to be used as a source of DNA for possible future molecular studies. Nails were clipped from the fingers and toes and about $0.5 \mathrm{~g}$ of the individual's hair was cut for future assessment of trace elements and toxicological studies deemed important in gastric carcinogenesis.

The participants then underwent standard upper GI video endoscopy under sedation with Midazolam (5-7.5 mg slow intravenous push) under monitoring and local anaesthesia of pharyngeal mucosa with $10 \%$ Lidocaine spray. A list of the endoscopic findings, using a special coding system for different lesions and a special endoscopy report form, were used to record the visual findings and generate an endoscopy report for future clinical decisions for the participant if necessary. The gastroenterologists in charge of the endoscopy procedure were assisted by trained personnel and general practitioners during the endoscopy and documentation of the endoscopic findings. Images of all the abnormal findings in the oesophagus, stomach, and duodenum were captured. Gastric biopsies were taken from the following sites:

- Incisura angularis for rapid urease test (first biopsy).
- Antrum, two biopsies, one between the incisura angularis and pyloric canal and the other on the opposite side over the greater curvature.

- Cardia, two biopsies 0.5 to $1.0 \mathrm{~cm}$ below the gastrooesophageal junction on the anterior and posterior gastric wall.

- All the visible lesions (number of biopsies and sites decided according to the endoscopic features noted by the gastroenterologist).

In addition to the above biopsies, two more biopsies from the corpus at greater and lesser curvature were obtained in 508 individuals studied in Meshkinshahr.

All the biopsies were received by trained pathology assistants present in the endoscopy room, who flattened and oriented the samples by the muscularis mucosa side over small pieces of filter paper. These samples were immediately and completely submerged in neutral buffered formalin in clearly labelled containers. Records of the numbers and sites of the biopsies were kept using special forms and a coding system. At the end of the procedure, the data recorded on the endoscopy and pathology forms were rechecked to ensure that they were correct. The results of the rapid urease test were documented on both forms as soon as available. The pathologist in charge supervised the process of tissue orientation, fixation, labelling, and recording.

After the endoscopy, the participants were transferred to the recovery room to be kept under close observation, with control of vital signs, until they had completely recovered from sedation. The individuals were then discharged to be returned to their residential area or taken to the gastroenterologist so that further clinical measures or treatments could be decided upon if any abnormality was noted during the endoscopy. Appropriate medications were given free of charge if necessary.

All the tissue samples were transferred to the DDRC research pathology laboratory in Tehran, processed, and embedded in paraffin wax blocks, according to standard protocols. Histological sections were stained by the haematoxylin and eosin method.

The histological sections were reviewed by three pathologists with a special interest and experience in GI pathology; they were informed of the site of each biopsy, but had no knowledge of the endoscopic and clinical findings. Special forms were used to record the pathological findings. All the diagnostic criteria used for our survey were discussed and sample slides were reviewed by the pathologists before the study was undertaken to minimise interobserver variations as much as possible. For the sake of quality assurance, 1/10 of the samples were randomly checked by another pathologist in the team, and all the cases with crucial histological findings were reviewed and discussed in joint sessions.

\section{HISTOLOGICAL CRITERIA AND DEFINITIONS Gastritis}

Gastritis is defined as the presence of increased numbers of inflammatory cells of any kind in the lamina propria. However, a few scattered lymphocytes and rare plasma cells can normally be seen and tolerated in the normal gastric antral and cardiac mucosa. Active chronic gastritis is defined as increased numbers of lymphocytes and plasma cells (chronic gastritis), accompanied by polymorphonuclear leucocytes. These polymorphonuclear leucocytes may show evidence of glandular invasion, similar to the crypt abscesses seen in inflammatory bowel diseases. ${ }^{14}$

\section{Mucosal atrophy}

Mucosal atrophy is defined as the separation of the mucosal glands and a decrease in the thickness of mucosa, greater in 
severity than that seen in inflammation of the lamina propria, and usually associated with an increase in the stromal matrix. ${ }^{14}$

\section{Intestinal metaplasia}

Intestinal metaplasia (IM) is defined as metaplastic transformation of gastric glandular and surface epithelium towards intestinal mucosal elements including goblet cells, absorptive cells, and Paneth cells. The presence of definite goblet cells is the minimum requirement for the histological diagnosis of IM. ${ }^{14}$

\section{Dysplasia}

Dysplasia is defined as unequivocal histological evidence of neoplastic transformation of epithelial cells, including a constellation of enlargement, hyperchromasia, irregularity, and pleomorphism of nuclei, nuclear roundening and loss of polarity, increased mitotic activity with abnormal figures, decreased cellular mucin content, crowding, and stratification of the epithelial cells. These changes should be limited to the epithelium confined to the intact basement membrane, with no evidence of invasion to the lamina propria or surrounding tissue reaction (desmoplasia). The severity (grade) of dysplasia depends on the intensity and extent of the involvement of the glandular and surface epithelium of the gastric mucosa, and is usually classified as low or high grade. $^{14} 15$

\section{Reactive atypia}

Reactive atypia is defined as cytological and histological changes similar to low grade dysplasia with coexistence of active inflammation and/or ulceration. This lesion is also called indefinite for dysplasia in some classifications. ${ }^{16}$ To avoid misclassification we used the term reactive atypia instead of low grade dysplasia or indefinite for dysplasia.

\section{Adenocarcinoma}

Adenocarcinoma is diagnosed when the malignant cells invade the lamina propria in single cell, glandular, or solid nest arrangements, usually accompanied by fibrosis of the surrounding tissue (desmoplasia). ${ }^{14} 16$

\section{Statistical analysis}

The data were expressed as mean (SD), and 95\% confidence intervals were calculated for all point prevalences of the histological findings. The presence of a variable (for example, active chronic gastritis) in at least one biopsy sample from the antral site was considered a positive finding. The absence of abnormal findings in all specimens from the same biopsy site was considered as normal. All data were analysed using SPSS10.0 for Windows.

\section{RESULTS}

One thousand and eleven individuals, or $91.5 \%$ of those found eligible, accepted the invitation to participate in our study: $494(48.9 \%)$ men and $517(51.1 \%)$ women. The youngest participant was 40 and the oldest 92 years old (mean age, 53.32; SD, 10.39). Five hundred and seven (50.1\%) participants were residents of the Ardabil and Meshkinshahr cities and the remaining 505 (49.9\%) were from rural areas. Except for one case of temporary respiratory depression following Midazolam, which was reversed promptly, all participants tolerated complete oesophagogastro-duodenoscopy uneventfully. Table 1 summarises the life styles, dietary habits, and body weights of the individuals investigated.

Endoscopy was reported to be normal in 198 (19.6\%) of the subjects. Table 2 summarises the important endoscopic findings. Nine hundred and eighty three (97.2\%) antral and
Table 1 Dietary habits, life style, and nutritional status

\begin{tabular}{|c|c|c|}
\hline Findings & $\mathrm{N}$ & $\%$ \\
\hline \multicolumn{3}{|c|}{ Meat consumption $(n=1008)$} \\
\hline Less than once a week & 229 & 22.7 \\
\hline $1-4$ times a week & 598 & 59.3 \\
\hline 5-7 times a week & 174 & 17.7 \\
\hline \multicolumn{3}{|l|}{ Fresh fruit $(n=1007)$} \\
\hline Less than once a week & 244 & 34.5 \\
\hline $1-4$ times a week & 490 & 48.7 \\
\hline 5-7 times a week & 196 & 16.8 \\
\hline \multicolumn{3}{|l|}{ Fresh vegetables $(n=1006)$} \\
\hline Less than once a week & 347 & 56.3 \\
\hline $1-4$ times a week & 156 & 31.0 \\
\hline 5-7 times a week & 64 & 12.7 \\
\hline \multicolumn{3}{|l|}{ Staple diet $(n=1007)$} \\
\hline Wheat & 847 & 84.1 \\
\hline Rice & 149 & 14.8 \\
\hline Other & 11 & 1.1 \\
\hline \multicolumn{3}{|l|}{ Habits $(n=1011)$} \\
\hline Tobacco smoking & 302 & 29.9 \\
\hline Opium smoking & 18 & 1.8 \\
\hline Alcohol consumption & 35 & 3.5 \\
\hline \multicolumn{3}{|l|}{ Body mass index (BMI) } \\
\hline \multicolumn{3}{|l|}{ Mean (SD), 27.1 (4.7) } \\
\hline $\mathrm{BMI} \leqslant 20$ & 25 & 3.4 \\
\hline $20<B M I \leqslant 25$ & 244 & 33.4 \\
\hline $25<\mathrm{BMI} \leqslant 30$ & 290 & 39.7 \\
\hline $30<\mathrm{BMI} \leqslant 35$ & 121 & 16.6 \\
\hline $\mathrm{BMl}>35$ & 51 & 7.0 \\
\hline \multicolumn{3}{|c|}{ Digestive symptoms (>once/month) } \\
\hline Pyrosis & 535 & 52.9 \\
\hline Epigastric pain & 443 & 43.8 \\
\hline
\end{tabular}

$816(80.7 \%)$ cardia mucosal biopsies were considered satisfactory on histopathological examination. The number of satisfactory tissue samples from the corpus was 505 of 508 biopsies performed $(99.4 \%)$. The main causes of deferral of samples for pathological diagnoses were inadequate specimens as a result of small biopsies and lack of proper orientation of the tissue $(2.8 \%$ of antral, $0.6 \%$ of corpus, and $5.6 \%$ of cardia biopsy specimens). One hundred and twelve (13.7\%) of the attempted cardia biopsies showed only squamous oesophageal mucosa. This is because it is technically difficult to biopsy this site, resulting in more sampling errors compared with the corpus and antrum.

The rapid urease test and/or histology were reported to be positive in 883 of $990(89.2 \%)$ subjects in whom the tests were performed. Table 3 summarises the histopathological findings. At the cardiac mucosa, 306 of 816 subjects $(37.5 \%)$ had atrophic gastritis, IM, reactive atypia, or glandular dysplasia. These findings were present in 262 of 505

Table 2 Endoscopic findings

\begin{tabular}{lcc}
\hline & \multicolumn{2}{c}{ Total $(\mathbf{n}=\mathbf{1 0 1 1})$} \\
\cline { 2 - 3 } Finding & $\mathbf{N}$ & $\%$ \\
\hline Stomach & 688 & 68.1 \\
Erythema & 107 & 10.6 \\
Erosion & 3 & 0.3 \\
Friability & 48 & 4.7 \\
Nodularity & 9 & 0.9 \\
Polyp & 30 & 3.0 \\
Ulcer gastric & 19 & 1.9 \\
Ulcer (duodenal) & 2 & 0.2 \\
Atrophic mucosa & 3 & 0.3 \\
Raised/thickened area & 198 & 19.6 \\
Normal & & 27.7 \\
Oesophagus & 280 & 7.0 \\
GERD A & 71 & \\
GERD B, C, D &
\end{tabular}

GERD, gastro-oesophageal reflux disease. 
Table 3 Histopathological findings of gastric cardia, corpus, and antral biopsies

\begin{tabular}{|c|c|c|c|c|c|c|}
\hline \multirow[b]{2}{*}{ Histological findings } & \multicolumn{2}{|c|}{ Antrum (983) } & \multicolumn{2}{|c|}{ Corpus (505) } & \multicolumn{2}{|c|}{ Cardia (816) } \\
\hline & $\mathbf{N}$ & $\%(95 \% \mathrm{Cl})$ & $\mathbf{N}$ & $\%(95 \% \mathrm{Cl})$ & $\mathbf{N}$ & $\%(95 \% \mathrm{Cl})$ \\
\hline Normal & 30 & 3.1 (2.0 to 4.2$)$ & 37 & 7.3 (5.0 to 9.6$)$ & 111 & $13.6(11.2$ to 16.0$)$ \\
\hline Chronic gastritis & 338 & 34.4 (31.4 to 37.4$)$ & 271 & 53.7 (49.3 to 58.1$)$ & 224 & 27.5 (24.4 to 30.6$)$ \\
\hline Active chronic gastritis & 765 & $77.8(75.2$ to 80.4$)$ & 303 & $60.0(55.7$ to 64.3$)$ & 472 & $57.8(54.4$ to 61.2$)$ \\
\hline H pylori infection & 818 & $83.2(80.9$ to 85.5$)$ & 328 & $65.0(60.8$ to 69.2$)$ & 492 & 60.3 (56.9 to 63.7$)$ \\
\hline Reactive atypia & 431 & 43.8 (40.7 to 46.9$)$ & 109 & 21.6 (18.0 to 25.2$)$ & 187 & 22.9 (21.3 to 24.5$)$ \\
\hline Atrophic changes & 444 & 45.2 (42.1 to 48.3$)$ & 232 & 46.9 (42.6 to 51.2$)$ & 179 & $21.9(20.5$ to 23.3$)$ \\
\hline Intestinal metaplasia & 128 & $13.0(10.9$ to 15.1$)$ & 42 & $8.3(5.9$ to 10.7$)$ & 31 & $3.8(2.5$ to 5.1$)$ \\
\hline Glandular dysplasia & 2 & $0.20(-0.07$ to 0.47$)$ & 1 & $0.2(0.01$ to 0.39$)$ & 2 & $0.25(-0.09$ to 0.58$)$ \\
\hline Adenocarcinoma & 3 & $0.31(-0.03$ to 0.65$)$ & 0 & 0 & 1 & $0.12(-0.11$ to 0.35$)$ \\
\hline
\end{tabular}

Antrum: positive finding in at least one of $\mathrm{B} \times 1, \mathrm{~B} \times 2$, or $\mathrm{B} \times 3$; corpus: positive finding in at least one of $\mathrm{B} \times 4$ or $\mathrm{B} \times 5$.

$\mathrm{Cl}$, confidence interval.

(51.9\%) of corporal biopsies and in 650 of $983(66.1 \%)$ of the antral biopsies. Table 4 shows the distribution of atrophy at different biopsy sites of the gastric mucosa in 508 cases from Meshkinshahr.

Statistical analysis (table 5) revealed no significant correlation between demographic factors and precancerous lesions, but it did show a protective effect of meat ( $\mathrm{p}$ for trend $<0.05$ ) and fresh vegetable ( $p$ for trend $<0.01$ ) consumption for antral IM only, but not for corpus or cardia IM.

\section{DISCUSSION}

Gastric carcinogenesis involves a slow but continuous stepwise evolution from superficial gastritis, glandular atrophy to metaplasia, dysplasia and, finally, to adenocarcinoma. ${ }^{15} 17$ This slow process of carcinogenesis, which may well extend over decades, provides an excellent opportunity for early detection and intervention to prevent further progression or regression of the carcinogenic process. This is especially true because $H$ pylori (which is readily treatable) is known to be the main aetiological agent and initiating carcinogen. Uemura et al have already shown that in Japan all patients who developed gastric cancer were infected with $H$ pylori. ${ }^{11}$ This finding may mean that other aetiological factors alone are not sufficient to induce gastric cancer and that $H$ pylori plays a pivotal role in the process of gastric carcinogenesis.

In Ardabil, which has the highest mortality from gastric cancer in Iran, we carried out a cross sectional endoscopic screening study to look for precancerous gastric lesions, with the aim of designing an appropriate preventive and interventional strategy to decrease the mortality from this fatal cancer. We have identified a range of precancerous lesions, the most notable of which were chronic active $H$ pylori associated gastritis in at least one of the biopsy sites in more than $90 \%$ of subjects. Atrophic gastritis, reactive atypia, IM,

Table 4 Distribution of atrophy in 508 subjects from Meshkinshahr with corpus biopsies

\begin{tabular}{lllr}
\hline Site & N (\%) & Subsite & N (\%) \\
\hline Cardia $(n=492)$ & 142 & Cardia+antrum+corpus & $112(22.0 \%)$ \\
& $(28.9 \%)$ & $\begin{array}{l}\text { Cardia+antrum } \\
\text { Cardia+corpus }\end{array}$ & $13(2.6 \%)$ \\
& & Cardia only & $7(1.0 \%)$ \\
Corpus $(n=505)$ & 223 & Corpus+antrum+cardia & $112(22.0 \%)$ \\
& $(45.9 \%)$ & Corpus+antrum & $86(17.2 \%)$ \\
& & Corpus+cardia & $10(2.0 \%)$ \\
Antrum $(n=508)$ & 270 & Corpus only & $15(3.0 \%)$ \\
& $(53.1 \%)$ & Antrum+corpus+cardia & $112(22.0 \%)$ \\
& & Antrum+corpus & $86(17.2 \%)$ \\
& & Antrum+cardia & $13(2.6 \%)$ \\
& & & $59(11.6 \%)$ \\
\hline
\end{tabular}

and dysplasia were seen in at least one biopsy site in $71.8 \%$ of subjects. The rate of IM and dysplasia was not as high as that reported from China ${ }^{18}{ }^{19}$ or Columbia. ${ }^{20}$ The reasons for this discrepancy may be (1) the age standardised rate for gastric cancer in Ardabile is 50, which is lower than in Linqu in northeast China or the Narin $\sim 0$ region of Colombia, where it is around 70 and (2) to prevent misclassification, we classified the less advanced lesions of low grade dysplasia and indefinite for dysplasia as reactive atypia, rather than dysplasia. We used only the urease test and histology (haematoxylin and eosin staining) to look for $H$ pylori and in $89.2 \%$ of the subjects, at least one of the tests was positive.

If we had also used serology or the breath test, or a more specific stain, such as Giemsa, the rate of $H$ pylori positivity would probably have been higher, perhaps approaching 9598\%. It seems that in Ardabil almost all people 40 year old and above had $H$ pylori associated chronic gastritis involving the antrum, corpus, and cardia, which was more severe in the antrum and corpus and became less prominent in the cardia. In contrast to the high risk populations of Japan, China, and Columbia, where more than $60 \%$ of gastric cancers are located in the antrum, in Ardabil more than $60 \%$ of gastric cancers are located in the proximal stomach, mainly in the cardia. ${ }^{621}$

Adenocarcinoma of the gastric cardia has a different biological behaviour, with a worse outcome and a shorter survival compared with its distal counterpart. Although $H$ pylori infection is well established as the main risk factor for non-cardia gastric cancer, studies from the USA and Europe have provided some evidence that cagA+ strains may have an inverse relation with gastric cardia and oesophageal adenocarcinomas, ${ }^{22-25}$ but reports from high risk regions ${ }^{26}{ }^{27}$ of Asia emphasise that these strains may also be a risk factor for proximal gastric cancer. It remains to be determined what proportion of proximal gastric cancer in these high risk regions of the world is the result of non- $H$ pylori related factors.

\section{"The adoption of a Western type life style and exposure to some additional, unknown environmental carcinogens may be the reason for the increasing rate of cardia cancer in Ardabil"}

The diverging trend in the incidence of gastric cancer in these two subsites in Western countries points to a different aetiology. Exposure to environmental carcinogens (such as nitric oxide), ${ }^{28}$ overeating, obesity, ${ }^{29}$ and resultant hyperacidity in the cardia region, ${ }^{30}$ which are the consequences of life style changes, seem to be more important in the pathogenesis of cardia gastric cancer.

During the past two decades, the Ardabili residents, who were all infected with $H$ pylori during childhood, ${ }^{31}$ have 
Table 5 Important dietary factors in patients with and without intestinal metaplasia (IM) in different parts of the stomach

\begin{tabular}{|c|c|c|c|c|c|c|c|c|c|}
\hline & \multicolumn{3}{|l|}{ Antrum } & \multicolumn{3}{|l|}{ Corpus } & \multicolumn{3}{|l|}{ Cardia } \\
\hline & With IM & No IM & $p$ Value & With IM & No IM & $p$ Value & With IM & No IM & p Value \\
\hline Meat consumption & & & $<0.05$ & & & 0.16 & & & 0.07 \\
\hline$<1 /$ week & 48 & 181 & & 17 & 124 & & 13 & 172 & \\
\hline 1-4/week & 55 & 543 & & 19 & 270 & & 13 & 471 & \\
\hline 5-7/week & 25 & 131 & & 6 & 69 & & 5 & 142 & \\
\hline Fresh fruit & & & 0.27 & & & 0.50 & & & 0.22 \\
\hline$<1 /$ week & 52 & 290 & & 17 & 157 & & 14 & 267 & \\
\hline 1-4/week & 55 & 424 & & 18 & 228 & & 13 & 384 & \\
\hline 5-7/week & 21 & 143 & & 7 & 78 & & 4 & 134 & \\
\hline Raw vegetables & & & $<0.01$ & & & 0.17 & & & 0.13 \\
\hline$<1 /$ week & 81 & 460 & & 27 & 251 & & 21 & 428 & \\
\hline 1-4/week & 38 & 284 & & 12 & 154 & & 8 & 259 & \\
\hline $5-7 /$ week & 9 & 111 & & 3 & 58 & & 2 & 98 & \\
\hline
\end{tabular}

gradually adopted a Western pattern of life style. More than $60 \%$ are overweight, with a body mass index of more than 25 (table 1), and most have access to adequate numbers of calories, mainly from bread, fat, and meat, whereas 35\% and $24 \%$ still do not eat enough fresh vegetables and fruit, respectively (table 1). More than $50 \%$ have at least one episode of pyrosis each week and $35 \%$ of them were found to have evidence of at least mild erosive oesophagitis.

It seems plausible that although $H$ pylori infection probably plays a pivotal role in the initiation of the carcinogenic process in the antrum, the adoption of a Western type life style and exposure to some additional, unknown environmental carcinogens may be the reason for the increasing rate of cardia cancer in Ardabil. Although we found that fresh vegetable and meat consumption had a protective effect against antral IM (table 5), our study failed to demonstrate a significant correlation with other dietary or demographic factors because of the small numbers of subjects with cancer and precancerous lesions. Further studies specifically designed to look for these correlations should be performed, such as case control studies using food frequency questionnaires.

The interaction between $H$ pylori induced carcinogenesis and hyperacidity induced hyperproliferation of the proximal gastric mucosa is also an interesting area of investigation. It is probable that $H$ pylori infection does not play a permissive role, as it most likely does in non-cardia gastric cancer, but it may be considered an additive risk, especially in high risk areas of the world, where both $H$ pylori infection and gastric cancer are more common.

Previous studies have indicated that screening for the detection and treatment of $H$ pylori infection to prevent gastric cancer is potentially cost effective if at least 30\% of cancers attributable to $H$ pylori can be prevented. ${ }^{32}$ The results of reported interventional trails from Japan and Columbia are very encouraging, and favour the notion that the eradication of $H$ pylori infection is a promising option for the prevention of gastric cancer, at least in high risk regions of the world; therefore, non-cardia gastric cancer may in the future be considered as a largely preventable disease. ${ }^{11}{ }^{13}$

The role of dietary nitrate derived nitric oxide in the aetiology of gastro-oesophageal junction cancer in Scotland ${ }^{22}$ has recently been related to the excess use of nitrogenous fertilisers and associated increased dietary nitrate exposure. In Ardabil, like other parts of Iran, there has been more than a 15 fold increase in the use of chemical nitrogenous fertilisers. Ardabil Province has also some geographical similarities with other high incidence areas of gastric cancer, the so called volcanic countries, such as Costa Rica, Chile, and Japan, by being located near a silent volcano named Sabalan. Therefore, a similar environmental factor, such as

\section{Take home messages}

- Endoscopic screening for upper gastrointestinal diseases was feasible and well tolerated in Ardabil, Iran, a high risk region for gastric cancer

- Most subjects showed Helicobacter pylori infection

- Atrophic gastritis, reactive atypia, and intestinal metaplasia were common in the antrum, corpus, and cardia subsites

nitrous volcanic soils, may contribute to the higher incidence of upper GI cancer in this area. ${ }^{33}$

Further epidemiological studies need to be performed to evaluate the role of dietary nitrates and other carcinogens, which could be secreted in saliva and accumulate in the cardia, in the pathogenesis of gastric cardia cancer.

Epidemiological studies should also be performed in different ethnic subgroups of northern, central, and south Iran with different prevalence rate for tumours looking at all four major types of upper GI cancers, including cancers of the distal stomach and gastric cardia, in addition to adenocarcinoma and squamous cell carcinoma of the oesophagus.

Our study indicates that, in Ardabile, adequate planning and resources may make such studies feasible and economic. Our findings are relevant to populations with very high gastric cancer rates and a very high prevalence of $H$ pylori infection. They may be less relevant to populations with a low gastric cancer risk, with a low prevalence of gastric cancer.

\section{ACKNOWLEDGEMENT}

This study was supported by a grant from the Academy of Medical Sciences of the Islamic Republic of Iran. Our appreciation is expressed to all medical students of Islamic Azad University of Ardabil, health personnel of Ardabil district, and all the kind people of Ardabil who were the subjects of this survey for their collaboration, and to Dr S M Dawsey of the Cancer Prevention Studies Branch, National Cancer Institute, Bethesda, Maryland, for valuable suggestions and comments.

\section{Authors' affiliations}

R Malekzadeh, M Sotoudeh, M H Derakhshan, J Mikaeli, S Merat, A Yoonessi, M Tavangar, B Abedi Ardakani, R Sotoudehmanesh, A Pourshams, A Ali Asgari, S Doulatshahi, B Ziad Alizadeh,

S Mir Moomen, Digestive Disease Research Centre, Tehran University of Medical Science, Tehran, 14114, Iran

A Yazdanbod, S Arshi, A Madjidpoor, Ardabil University of Medical Science, Ardabil, Iran

D E Fleischer, Division of Gastroenterology and Hepatology, Mayo

Clinic, Scottsdale, Arizona 85259, USA 


\section{REFERENCES}

1 Correa PP, Haenszel W. Epidemiology of gastric cancer. In: Correa P, Haenszel W, eds. Epidemiology of cancer of the digestive tract, Vol. 2. Hague: Mattinus-Niiihoff, 1998:58-84.

2 In: Parkin DM, Mujir CS, Whelan SL, et al. Cancer incidence in five continents, Vol. 6. Lyon: IARC Scientific Publication, No. 120, 1992:865-70.

3 Nadim A, Noorai M. Cancers. In: Azizi F, Hatami H, Janghorbani M, eds. Epidemiology and control of common diseases in Iran, 1st ed. Tehran: Eshtiagh, 2000:216-17.

4 Naghavi M. Death report from 10 provinces in Iran, 1st ed. Tehran: Ministry of Health, 2000:384.

5 Sadjadi AR, Malekzadeh R, Derakhshan MH, et al. Cancer occurrence in Ardabile: results of a population based cancer registry from Iran. Int J Cancer 2003;107:113-18.

6 Yazdanbod A, Derakhshan MH, Arshi S, et al. Gastric cardia cancer; the most common type of upper gastrointestinal cancer in Ardabil, Iran: an endoscopy clinic experience. Arch Int Med 2001:4:76-9.

7 Pisani P, Parkin DM, Munoz N, et al. Cancer and infection: estimates of the attributable fraction in 1990. Cancer Epidemiol Biomarkers Prev 1997;6:387-400

8 Forman D, Webb PM. H. pylori and gastric cancer: the significance of the problem. In: Hunt RH, Tygat GN, eds. Helicobacter pylori. Basic mechanisms to clinical cure. Dordrecht (The Netherlands): Kluwer Academic Publishers, 1994: 156-62.

9 International Agency for Research on Cancer (IARC) Working Group. IARC monographs on the evaluation of carcinogenic risks to humans: schistosomes, liver flukes and Helicobacter pylori, Vol. 61. Lyon (France): IARC, 1994.

10 Watanabe $\mathrm{T}$, Tada $\mathrm{M}$, Nagai $\mathrm{H}$, et al. Helicobacter pylori infection induces gastric cancer in Mongolian gerbils. Gastroenterology 1998;115:642-8.

11 Uemura N, Okamoto S, Yamamoto S, et al. Helicobacter pylori infection and the development of gastric cancer. N Engl J Med 2001;345:784-8.

12 Buiatti E, Munoz N, Kato I, et al. Determinants of plasma anti-oxidant vitamin levels in a population at high risk for stomach cancer. Int J Cancer 1996;65:317-22.

13 Correa $\mathbf{P}$, Fontham E, Bravo JC, et al. Chemoprevention of gastric dysplasia: randomized trial of antioxidant supplements and anti-Helicobacter pylori therapy. J Natl Cancer Inst 2000;92:1881-8.

14 Dixon MF, Genta RM, Yardley JH, et al. Classification and grading of gastritis. The updated Sydney system. International workshop on the histopathology of gastritis, Houston 1994. Am J Surg Pathol 1996;20:1161-81.

15 Clouston AD. Timely topic: premalignant lesions associated with adenocarcinoma of the upper gastrointestinal tract. Pathology $2001 ; 33: 271-7$.

16 Schlemper RJ, Riddell RH, Kato YY, et al. The Vienna classification of gastrointestinal epithelial neoplasia. Gut 2000;47:251-5.
17 Correa P. Human gastric carcinogenesis: a multistep and multifactorial process - First American Cancer Society award lecture on cancer epidemiology and prevention. Cancer Res 1992;52:6735-40.

18 You WC, Blot WJ, Li JY, et al. Precancerous gastric lesions in a population at high risk of stomach cancer. Cancer Res 1993:53:1317-21.

19 Wei-ChengYoui, Ji-You LI, William J Blot, et al. Evolution of precancerous lesion in a rural Chinese population at high risk of gastric cancer. Int J Cancer 1999;83:615-19.

20 Correa P, Haenszel W, Cuello C, et al. Gastric precancerous process in a high risk population: cross-sectional studies. Cancer Res 1990;50:4731-6.

21 You WC, Blot WJ, Chang YS, et al. Comparison of the anatomic distribution of stomach cancer and precancerous gastric lesions. Jpn J Cancer Res 1992;83:1150-3.

22 Hansson LR, Engstrand L, Nyren O, et al. Prevalence of Helicobacter pylori infection in subtypes of gastric cancer. Gastroenterology 1995;109:885-8.

23 Martin-de-Argila C, Boixeda D, Redondo C, et al. Relation between histologic subtypes and location of gastric cancer and Helicobacter pylori. Scand J Gastroenterol 1997:32:303-7.

24 Chow WH, Blaser MJ, Blot WJ, et al. An inverse relation between cagA+ strains of Helicobacter pylori infection and risk of esophageal and gastric cardia adenocarcinoma. Cancer Res 1998;15:588-90.

25 Hansen S, Melby KK, Aase S, et al. Helicobacter pylori infection and risk of cardia cancer and non-cardia gastric cancer. A nested case-control study. Scand J Gastroenterol 1999;34:353-60.

26 Limburg PJ, Qiao YL, Mark SD, et al. Helicobacter pylori seropositivity and subsite specific gastric cancer risks in Linxian, China. J Natl Cancer Inst 2001;93:226-33.

27 Kikuchi S, Wada O, Nakajima T, et al. Serum anti Helicobacter antibody and gastric carcinoma among young adults. Research group on prevention of gastric carcinoma among young adults. Cancer 1995;75:2789-93.

28 lijima K, Henry E, Moriya A, et al. Dietary nitrate generates potentially mutagenic concentrations of nitric oxide at the gastroesophageal junction. Gastroenterology 2002;122:1248-57.

29 Calle EE, Rodriguez C, Walker-Thurmond K, et al. Overweight, obesity, and mortality from cancer in a prospectively studied cohort of U.S. adults. N Engl J Med 2003;348:1625-38.

30 Moriya A, Grant J, Mowat C, et al. In vitro studies indicate that acid catalysed generation of $\mathrm{N}$-nitrosocompounds from dietary nitrate will be maximal at the gastro-oesophageal junction and cardia. Scand J Gastroenterol 2002;37:253-61.

31 Mikaeli J, Malekzadeh R, Ziad-Alizadeh B, et al. Prevalence of Helicobacter pylori in two Iranian provinces with high and low incidence of gastric carcinoma. Archives of Iranian Medicine 2000;3:6-9.

32 Parsonnet J, Harris RA, Hack HM, et al. Modeling cost-effectiveness of Helicobacter pylori screening to prevent gastric cancer: a mandate for clinical trials. Lancet 1996;348:150-4.

33 Wallerstein C. Costa Rica tackles high rates of stomach cancer. BMJ $2001 ; 323: 1270$ 